\title{
ZEB1 is involved in regulating HER2-positive breast cancer radiosensitivity by controlling EMT
}

\author{
FUSHI HAN ${ }^{1}$, HUIYUAN ZHU ${ }^{2}$, JINMING XU $^{1}$, WEI SHUI ${ }^{1}$ and XIAOLING LU ${ }^{3}$ \\ ${ }^{1}$ Department of Imaging, Yangpu Hospital, Tongji University School of Medicine, Shanghai 200090; \\ ${ }^{2}$ Medical Imaging Division, Pulmonary Hospital Affiliated to Tongji University, Shanghai 200092; \\ ${ }^{3}$ Department of Radiology, Yangpu Hospital, Tongji University School of Medicine, Shanghai 200090, P.R. China
}

Received July 12, 2017; Accepted November 9, 2017

DOI: $10.3892 / 01.2018 .7906$

\begin{abstract}
Breast cancer is a malignant tumor that can rapidly progress to cancer for which there are limited therapeutic options. The aim of the present study was to examine the regulatory mechanism of human epidermal growth factor receptor 2 (HER2)-positive breast cancer radiosensitivity by comparing the protein expression levels of HER2 in breast cancer cell lines. Breast cancer cell line MCF-7 (low HER2 expression) and breast cancer cell lines ZR-7530 and SK-BR-3 (high HER2 expression) were selected for the present study. The cell lines were treated with shRNA and different doses of radiation $(2,4$ and $6 \mathrm{~Gy})$. Immunohistochemistry was performed on HER2-positive breast cancer tissues to detect the protein and mRNA expression levels of ZEB1 and HER2. A Transwell assay was performed to determine changes in the invasion capacity of cells under the different treatments. Western blot analysis was performed to detect the changes in ZEB1 and E-cadherin protein levels, and qPCR was performed to detect the mRNA and protein expression levels of HER2, ZEB1 and E-cadherin. Furthermore, immunohistochemistry was performed in tissues to detect the protein levels of HER2, ZEB1 and E-cadherin, and determine their correlation. The results showed that, ZEB1 protein and mRNA levels were higher in ZR-7530 and SK-BR-3 cells with a high HER2 expression compared to that in MCF-7 lines with a low HER2 expression. After $0,2,4$ and 6 Gy of radiation treatments, the cell invasion inhibitory rate in the no-load control group was $0.00,18.70,31.24$ and $47.66 \%$, respectively, while the cell invasion inhibitory rate in the shRNA group was $0.00,25.32$, 40.71 and $58.46 \%$, respectively. Compared with the no-load control group, the cell invasion inhibitory rate was higher in the treatment group, and the difference was statistically
\end{abstract}

Correspondence to: Dr Xiaoling Lu, Department of Radiology, Yangpu Hospital, Tongji University School of Medicine, 450 Tengyue Road, Shanghai 200090, P.R. China

E-mail: lug2ms@163.com

Key words: radiation sensitivity, EMT progression, HER2-positive breast cancer, ZEB1 significant $(\mathrm{P}<0.05)$. Furthermore, the protein and mRNA levels of E-cadherin increased after HER2 knockdown treatment. Based on the Student's t-test analysis, the difference was statistically significant $(\mathrm{P}<0.05)$. Pearson's analysis revealed that HER 2 protein levels were positively correlated with ZEB1 protein levels $(\mathrm{r}=0.480, \mathrm{P}=0.013)$, but was negatively correlated with $\mathrm{E}$-cadherin protein levels $(\mathrm{r}=-0.650, \mathrm{P}=0.000)$. Therefore, ZEB1 protein can affect HER2-positive breast cancer cell epithelial-mesenchymal transition progression, and is involved in regulating cell radiosensitivity. This finding suggests that ZEB1 is a potential target protein that can be used as a clinical inhibitor for changes in HER2-positive breast cancer radiation sensitivity.

\section{Introduction}

Breast cancer is a malignant tumor that influences the life and health of women worldwide, and which rapidly progresses to cancer $(1,2)$. Treatment and drugs are very limited at this stage. Radiotherapy for breast cancer is one of the main clinical applications currently available $(3,4)$. However, the majority of cancers have exhibited radiotherapy resistance, which seriously impacts the effect of such treatment $(5,6)$. Human epidermal growth factor receptor 2 (HER2) is widely expressed in various types of cancer, including gastric and prostate cancer $(7,8)$. HER2 protein overexpression has often been found in clinical breast cancer patients, and the clinical manifestations of such patients are exceptionally poor, including higher degrees of malignancy, strong metastatic ability and clinically low patient survival (9). In addition, it has been reported that radiation resistance often occurs when treating HER2-positive breast cancer patients in clinic (10), which greatly influences radiation therapy. However, at present, few studies have investigated the mechanism involved in the radiosensitivity of HER2-positive breast cancer patients. Therefore, it is imperative to enhance the radiosensitivity of patients with HER2-positive breast cancer.

Epithelial-mesenchymal transition (EMT) is a key step to malignant tumor transformation. When EMT occurs in cells, cell-to-cell adhesion is reduced, and cell invasion and transfer capabilities are increased (11). It has been previously reported that Chd1 genes that encode cell adhesion protein E-cadherin are involved in EMT, and the downregulation of this protein 
is one of the important characteristics of EMT (12). Similarly, Zinc Finger E-Box Binding Homeobox 1 (ZEB1) is a classical transcription factor protein that binds to the chd1 gene promoter region, recruits related inhibitory complexes, and apparently regulates the suppression of E-cadherin protein expression levels, thereby participating in EMT progression (13).

After reviewing relevant studies, it was found that the radiosensitivity regulation mechanism in HER2-positive breast cancer patients has not been studied in detail. Furthermore, EMT is a key step in cancer development, and is very important in breast cancer invasion and metastasis. Additionally, the investigators consider that the decrease in radiosensitivity in patients with HER2-positive breast cancer may be associated with the increase in EMT progression. Therefore, the protein expression levels of HER2 in different breast cancer cells were explored to determine the molecular mechanism of the reduced radiosensitivity of HER2-positive breast cancer cells, providing a potential target for radiation therapy in patients with HER2-positive breast cancer. The results showed that, ZEB1 is a potential target protein that can be used as a clinical inhibitor for changes in HER2-positive breast cancer radiation sensitivity.

\section{Materials and methods}

\section{Experimental materials}

Experimental cells. The 293T cells and human breast cancer lines MCF-7, ZR-7530 and SK-BR-3 were obtained from the Chinese Academy of Sciences Cell Bank (Shanghai, China). Approval for the study was obtained by the Ethics Committee of Yangpu Hospital (Shanghai, China).

Experimental reagents. RPMI-1640, fetal bovine serum (FBS), streptomycin and trypsin were purchased from Gibco; Thermo Fisher Scientific, Inc., (Waltham, MA, USA); IRES, PLKO.1 (puro resistance) viral vector, HER2 plasmid vector and ZEB1 plasmid vector were purchased from Addgene, Inc. (Cambridge, MA,USA); puroreagent, and Matrigel (both from Sigma-Aldrich; Merck KGaA, Darmstadt, Germany); DNA ligase, and RT-PCR kit were purchased from Takara Biotechnology Co., Ltd. (Dalian, China); DNA cleavage enzymes, qPCR kit, PCR and qPCR primers, TRIzol and the BCA protein concentration assay kit were purchased from Thermo Fisher Scientific, Inc.; ZEB1, HER2, E-cadherin, and GAPDH proteins were purchased from Abcam (Cambridge, UK); horseradish peroxidase-labeled rabbit, and mouse secondary antibodies were purchased from Santa Cruz Biotechnology, Inc. (Dallas, TX, USA); and ECL color developing solution was purchased from Beyotime Institute of Biotechnology (Shanghai, China).

Experimental apparatus. Western blotting instruments and PCR instruments were purchased from Bio-Rad Laboratories, Inc. (Hercules, CA, USA). A quantitative PCR system (ABI7500; Applied Biosystems; Thermo Fisher Scientific, Inc.); inverted, and immunofluorescence microscopes (Olympus Corporation, Tokyo, Japan); and incubator, and refrigerated centrifuge (Thermo Fisher Scientific, Inc.) were used in the present study.

Cell culture. Cells were cultured in RPMI-1640 medium containing $10 \% \mathrm{FBS}$ with $5 \% \mathrm{CO}_{2}$ at $37^{\circ} \mathrm{C}$, until the cells reached $80 \%$ confluence. Passaged cells were digested with $0.25 \%$ trypsin, and cultured until the logarithmic growth phase was reached.

Establishment of stably transfected cell lines. The synthesis of ZEB1 protein shRNA up- and downstream primers was performed, and the ZEB1-shRNA virus vector was constructed through gene recombination. The viral vector was expressed in $293 \mathrm{~T}$ cells, the supernatant was collected once every $24 \mathrm{~h}$, the liquid was changed, collected twice, and the virus was filtered and stored at $4^{\circ} \mathrm{C}$. Human breast cancer cells were infected with the virus. After $48 \mathrm{~h}$, dose screening was performed on stably transfected cells. In the blank control group, all the cells died. The establishment of stable cell lines was completed, passaging was tested, and the protein and mRNA levels were detected.

Cell radiation treatment. The cells were vertically irradiated from the bottom of the culture plate for $3 \mathrm{~min}$ at irradiation doses of 2,4 and $6 \mathrm{~Gy}$, respectively.

Transwell invasion experiment. Precooled serum-free medium was diluted with Matrigel glue, spread to the upper chamber, and placed in a cell incubator for 2-4 h. Human breast cancer cells diluted in serum-free medium were inoculated, and $500 \mu \mathrm{l}$ of serum-containing medium was added to the lower chamber. After $18 \mathrm{~h}$ incubation, crystal violet staining was performed for $20 \mathrm{~min}$. After washing, the cells were observed under an inverted microscope and counted. Three wells were set for each group, and the cells were counted in five fields. The invasion inhibition rate (\%) was calculated as: $(1$ - number of cells in the invasion group/number of cells in control group) x100\%

Western blot analysis. When the cells grew to the logarithmic phase, they were digested by trypsin, washed with phosphate-buffered saline (PBS) three times, added with an appropriate amount of lysis buffer, blown evenly, cleavaged for $30 \mathrm{~min}$, and the protein supernatant was collected for BCA protein quantification.

BCA quantitative results revealed that the adjusted protein concentration of each sample was consistent. The sample was loaded and heated at $95^{\circ} \mathrm{C}$ for $10 \mathrm{~min}$, and frozen at $-20^{\circ} \mathrm{C}$ for later use. The samples were centrifuged for $3 \mathrm{~min}$ at $2,600 \mathrm{x} \mathrm{g}$ prior to loading, blown evenly, and electrophoresis was carried out at a constant voltage of $90 \mathrm{~V}$ for approximately $120 \mathrm{~min}$, with a constant current of $400 \mathrm{~mA}$ on the film for $90 \mathrm{~min}$. The membrane was blocked with $5 \%$ skim milk for $1 \mathrm{~h}$, and the samples were incubated with goat anti-human ZEB1 polyclonal antibody (1:500; cat. no. c-10572) overnight at $4^{\circ} \mathrm{C}$. The following day, the samples were incubated with rabbit anti goat secondary polyclonal antibody (1:1000; cat. no. sc-2768) at room temperature for $1 \mathrm{~h}$, and exposure treatment was performed with GAPDH protein as the internal reference.

$q P C R$. When the cells grew to approximately $80 \%$ confluence, TRIzol reagent was added to lyse the cells. The lysate was transferred into a centrifuge tube, and chloroform was added. After mixing well, the cells were centrifuged for $10 \mathrm{~min}$ at $8,600 \times \mathrm{g}$. Subsequently, the upper RNA was transferred into a new centrifuge tube, and the same volume of isopropanol was added. After mixing well, the cells were centrifuged 
Table I. qPCR primer sequences.

\begin{tabular}{lll}
\hline Gene & \multicolumn{1}{c}{ 5' sequence } & \multicolumn{1}{c}{ 3' sequence } \\
\hline HER 2 & CCCATATGTCTCCCGCCTTC & GGTTTTCCCGGACATGGTCT \\
$Z E B 1$ & CAGCTTGATACCTGTGAATGGG & TATCTGTGGTCGTGTGGGACT \\
E-cadherin & GAAATCACATCCTACACTGCCC & GTAGCAACTGGAGAACCATTGTC \\
GAPDH & ACCAAATCCGTTGACTCCGACCTT & TCGACAGTCAGCCGCATCTTCTTT \\
\hline
\end{tabular}

HER2, human epidermal growth factor receptor 2; GAPDH, glyceraldehyde-3-phosphate dehydrogenase; qPCR, quantitative polymerase chain reaction.


Figure 1. Comparison of HER2 and ZEB1 protein mRNA levels. (A) HER2 and (B) ZEB1 protein mRNA levels were decreased in MCF-7 cells compared to the other breast cancer cell lines. ${ }^{* *} \mathrm{P}<0.01$, compared with the MCF-7 cell line. HER2, human epidermal growth factor receptor 2.

at maximum speed. The supernatant was discarded, and the precipitate was washed with $75 \%$ ethanol. After drying in the fume hood, nuclease-free water was added at $70^{\circ} \mathrm{C}$, dissolved for $10 \mathrm{~min}$, and stored at $-80^{\circ} \mathrm{C}$ for later use. RNA $(1 \mu \mathrm{g})$ was taken for reverse transcription reaction to synthesize cDNA. This was performed in triplicate for each sample set on the machine. Data processing was performed using the $\Delta \Delta \mathrm{Cq}$ method, and relative protein mRNA expression levels were compared. The qPCR primers designed in the experiment are presented in Table I.

Immunohistochemistry. Tissue specimens were fixed in $10 \%$ formalin and paraffin-embedded. The slides were dyed (H\&E staining) after being dried in an oven. For dewaxing, the slides were immersed in xylene solutions I and II, respectively, for $10 \mathrm{~min}$. The slides were then immersed separately in 100 , 95 and $75 \%$ alcohol, soaked for $5 \mathrm{~min}$, rinsed with PBS, and dried. For antigen retrieval, the repair fluid was heated until boiling point, after which the slides were immersed in the heated fluid for approximately $2 \mathrm{~min}$, cooled and rinsed. For inactivation, the slides were immersed in hydrogen peroxide solution, incubated at room temperature for $10 \mathrm{~min}$, removed and rinsed. The primary antibody was then dripped on the slides, which were agitated overnight at $4^{\circ} \mathrm{C}$. After the slides were rinsed, polymer enhancers were dripped, and incubated for $20 \mathrm{~min}$. After rinsing, the slides were again incubated at room temperature for $30 \mathrm{~min}$. DAB was dripped on the slides and observed under a microscope. The staining process was stopped when the color turned to tan brown or brown. The slides were dyed with hematoxylin, underwent acidification, and returned to blue. Following gradient alcohol dehydration, the slides were mounted with neutral gum.

Statistical analysis. Data in the present study were analyzed using SPSS 19.0 statistical software (SPSS, Inc., Chicago, IL, USA). Measurement data were presented as mean \pm standard deviation. Comparison between two samples was performed using the ANOVA and Student-Newman-Keuls post-hoc test. Correlation analysis was performed using the Pearson's correlation coefficient test. $\mathrm{P}<0.05$ was considered to indicate a statistically significant analysis.

\section{Results}

Comparison of HER2 and ZEB1 protein mRNAs in different human breast cancer cell lines. qPCR results revealed that HER2 and ZEB1 protein mRNA levels were lower in MCF-7 cells than in ZR-7530 and SK-BR-3 cells. The independent samples t-test analysis revealed that the difference was statistically significant $(\mathrm{P}<0.05$, Fig. 1$)$.

Detection of ZEB1 protein knockdown efficiency. The results of the western blot analysis revealed that there was a small amount of ZEB1 protein in the shRNA group, while the expression level of ZEB1 protein in the no-load control 
group was higher, and the difference was statistically significant $(\mathrm{P}<0.05)$ (Fig. 2). At the same time, qPCR showed that compared with the no-load control group, ZEB1 protein and mRNA levels decreased in the shRNA group and through t-test analysis, the difference was found to be statistically significant $(\mathrm{P}<0.05$; Fig. $1 \mathrm{~A}$ and $\mathrm{B})$.

Effect of knockdown of ZEB1 protein on SK-BR-3 cell invasion inhibition rate. After the knockdown of ZEB1 and treatment with 2, 4 and 6 Gy of irradiation, the SK-BR-3 cell invasion rate increased, compared with the no-load group. The independent samples t-test analysis revealed that the difference was statistically significant $(\mathrm{P}<0.05$, Fig. 3$)$.

Effect of knockdown of ZEB1 protein on E-cadherin protein in SK-BR-3 cells. After 0,2,4 and 6 Gy of radiation treatment, E-cadherin protein and mRNA levels in the cells increased. Compared with the no-load control group, E-cadherin protein and mRNA levels were higher in the shRNA group. The independent samples t-test analysis revealed that the difference was statistically significant $(\mathrm{P}<0.05$, Fig. 4).

Detection of HER2, ZEB1 and E-cadherin protein levels in HER2-positive breast cancer specimens by immunohistochemistry. Pearson's correlation coefficient revealed that HER2 and ZEB1 protein levels were positively correlated $(\mathrm{r}=0.480)$, and the difference was statistically significant $(\mathrm{P}<0.05)$. However, these were negatively correlated with E-cadherin levels ( $\mathrm{r}=-0.650)$, and the difference was statistically significant $(\mathrm{P}<0.05)$ (Fig. 5).

\section{Discussion}

Breast cancer is a common major cancer in women, and its morbidity and mortality continue to increase (14), posing a threat to the lives and health of women. From a genetic point of view, breast cancer can be divided into two categories: Estrogen-positive and estrogen-negative breast cancer. For estrogen-negative breast cancer, this can be further divided into HER2-positive breast cancer and normal breast cancer (15). Of these, HER2-positive breast cancer rapidly progresses in patients, has poor prognosis, and is subject to clinical attention $(16,17)$. In addition, radiation sensitivity in HER2-positive breast cancer patients is also a major challenge in clinic (18).

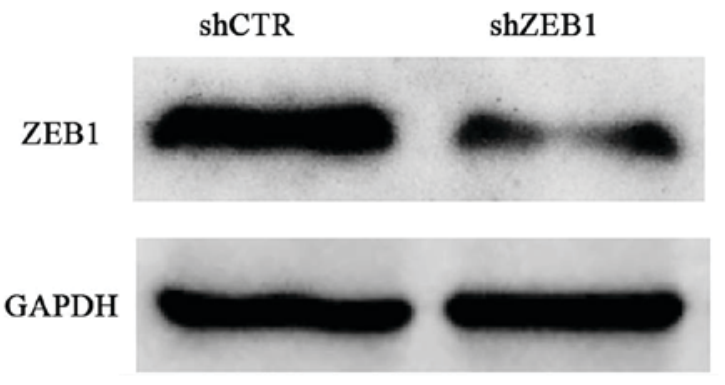

Figure 2. Detection of knockdown of ZEB1 protein.

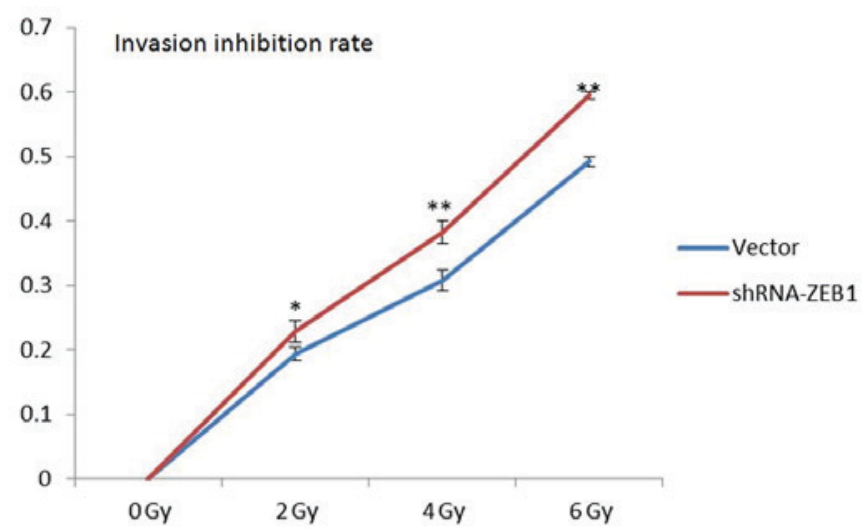

Figure 3. Effect of knockdown of ZEB1 protein on SK-BR-3 cell invasion inhibition rate. ${ }^{*} \mathrm{P}<0.05$, compared with the no-load control group; ${ }^{* *} \mathrm{P}<0.01$, compared with the no-load control group.

Thus, the investigators detected the protein levels of breast cancer cell lines with different HER2 expression levels, determined the different transcription factor ZEB1 expression levels, and explored the role of ZEB1 protein in HER2-positive breast cancer radiosensitivity.

Results of the present study confirm that the protein expression levels of transcription factor ZEB1 were higher in breast cancer cell lines with a high HER 2 expression than in cell lines with a low HER2 expression, suggesting that ZEB1 protein levels may play a key role in HER2-positive breast cancer cell lines. Previous findings have indicated thatZEB1/2 transcription
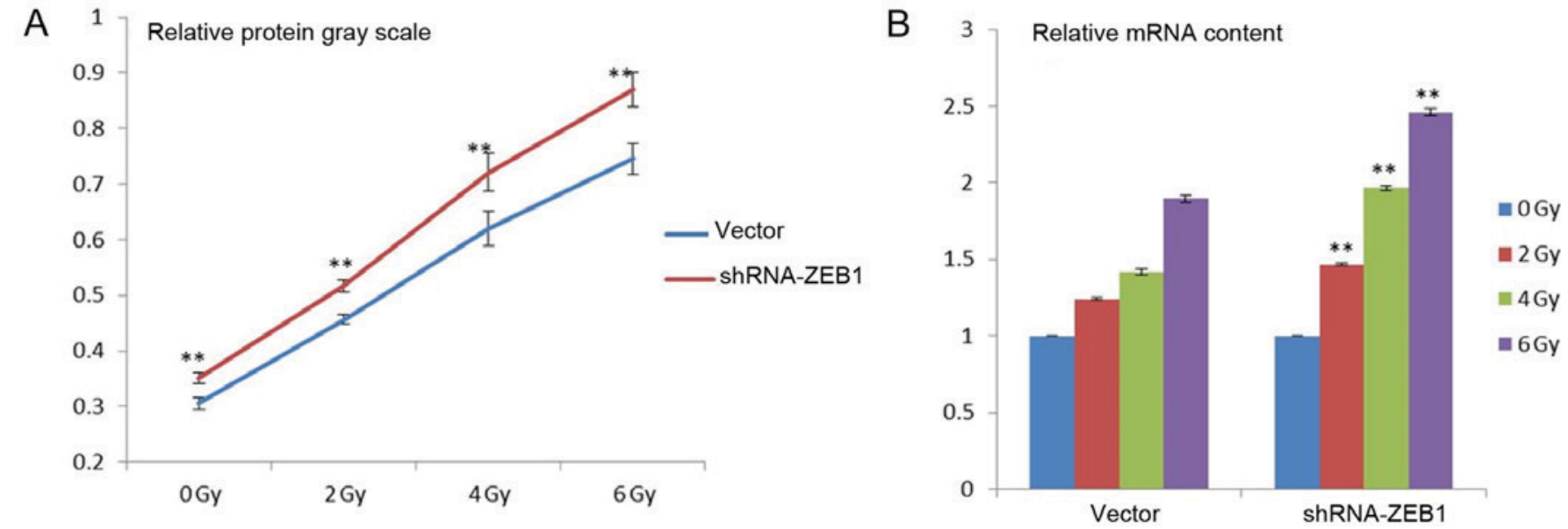

Figure 4. (A) Effect of knockdown of ZEB1 protein on E-cadherin protein levels. ${ }^{* *} \mathrm{P}<0.01$, compared with the no-load control group. (B) Effect of knockdown of ZEB1 on E-cadherin mRNA levels. ${ }^{* *} \mathrm{P}<0.01$, compared with the no-load control group. 

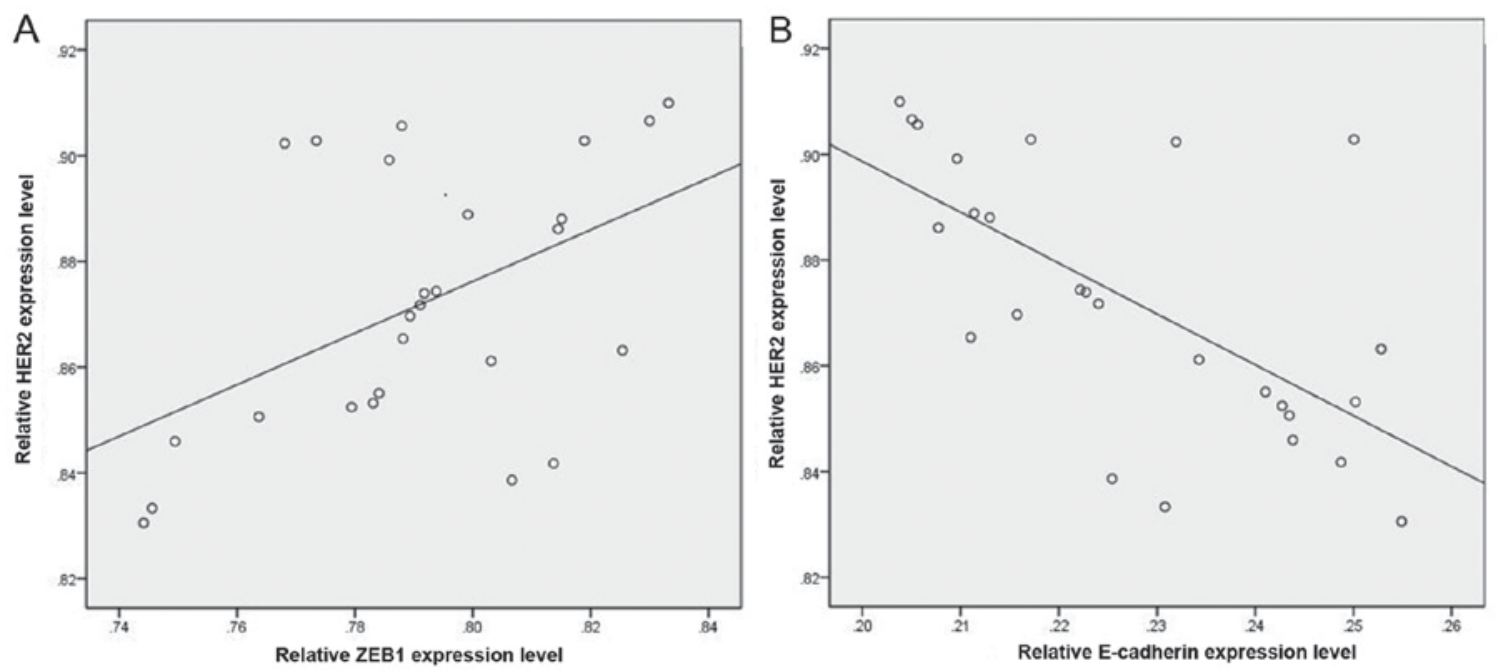

Figure 5. Correlation of (A) HER2 and ZEB1 and (B) HER2 and E-cadherin protein expression levels. HER2, human epidermal growth factor receptor 2.

factor protein levels play a key role in cancer epidermal stroma transformation $(19,20)$. However, reports on whether ZEB1 protein participates and regulates HER2-positive breast cancer radiosensitivity remains to be determined. Therefore, we knocked down the protein expression of ZEB1, combined this with radiation treatment, and observed the changes in cell invasion capability.

Results of the present study have shown that with increasing radiation dose, the invasion inhibition rate of SK-BR-3 cells gradually increased Following further ZEB1 protein knockdown, the change in the invasion inhibition rate was more obvious. In addition, for each radiation dose and after ZEB1 knockdown, the invasion inhibition rate was higher than that in the control group, suggesting that $\mathrm{ZEB} 1$ protein may be involved in the regulation of the radiosensitivity of SK-BR-3 cells with a high HER2 expression. Additionally, the investigators detected the E-cadherin marker protein during EMT, and found that E-cadherin protein levels increased with the elevation in radiation dose. In addition, E-cadherin protein levels more significantly increased in the shRNA treatment group compared with the no-load control group, further suggesting that ZEB1 is involved in the regulation of cell radiosensitivity, and may affect the radiosensitivity of cells by controlling the progression of cell EMT.

Immunohistochemical results on HER2-positive breast cancer clinical specimens revealed that the protein expression of HER2 and ZEB1 was positively correlated, which is consistent with results of the present study in the detection of cell lines. In addition, the protein expression of HER2 and E-cadherin was negatively correlated, suggesting that HER2-positive breast cancer patients had a higher cancer cell invasion ability, and were prone to chemotherapy resistance. Furthermore, the results suggest that $\mathrm{ZEB} 1$ protein is involved in the regulation of radiosensitivity in HER2-positive breast cancer patients, and that this regulation process is affected by the impact of E-cadherin protein in EMT progression.

However, a number of questions remain to be confirmed. First, there is a need to further verify whether ZEB1 protein regulates the protein expression of E-cadherin by transcription. In addition, regarding the involvement of ZEB1 protein in the radiosensitivity upstream regulatory pathway of patients with overexpressed HER2, further studies are required to determine how radiation affects the protein level of ZEB1. In summary, the results of the present study confirm that ZEB1 may be involved in the radiosensitivity of HER2-positive breast cancer by regulating EMT progression, providing a potential molecular target for enhancing the radiosensitivity of these patients.

\section{Acknowledgements}

Not applicable.

\section{Funding}

No funding was received.

\section{Availability of data and materials}

The datasets used and/or analyzed during the current study are available from the corresponding author on reasonable request.

\section{Authors' contributions}

$\mathrm{FH}, \mathrm{HZ}$ and JX designed the research to examine the regulatory mechanism of HER2-positive breast cancer radiosensitivity and FH was a major contributor in writing the manuscript. WS and XL performed the research and analyzed the data. All authors read and approved the final manuscript.

\section{Ethics approval and consent to participate}

Approval for the study was obtained by the Ethics Committee of Yangpu Hospital (Shanghai, China).

\section{Consent for publication}

Not applicable.

\section{Competing interests}

The authors declare that they have no competing interests. 


\section{References}

1. Thomson CA and Thompson PA: Dietary patterns, risk and prognosis of breast cancer. Future Oncol 5: 1257-1269, 2009.

2. Fusco P, Scimia P, Petrucci E, DI Carlo S and Marinangeli F: The ultrasound-guided parasternal block: A novel approach for anesthesia and analgesia in breast cancer surgery. Minerva Anestesiol 83: 221-222, 2017.

3. Herskind C, Steil V, Kraus-Tiefenbacher U and Wenz F: Radiobiological aspects of intraoperative radiotherapy (IORT) with isotropic low-energy $\mathrm{X}$ rays for early-stage breast cancer. Radiat Res 163: 208-215, 2005.

4. McGale P, Taylor C, Correa C, Cutter D, Duane F, Ewertz M, Gray R, Mannu G, Peto R, Whelan T, et al: EBCTCG (Early Breast Cancer Trialist's Collaborative Group): Effect of radiotherapy after mastectomy and axillary surgery on 10-year recurrence and 20-year breast cancer mortality: Meta-analysis of individual patient data for 8135 women in 22 randomised trials. Lancet 383: 2127-2135, 2014

5. Li Z, Yang X, Xia N, Yang L, Yu H, Zhou F, X C and Zhou Y: PTOP and TRF1 help enhance the radio resistance in breast cancer cell. Cancer Cell Int 14: 7, 2014.

6. Duru N, Fan M, Candas D, Menaa C, Liu HC, Nantajit D, Wen Y, Xiao K, Eldridge A, Chromy BA, et al: HER2-associated radioresistance of breast cancer stem cells isolated from HER2-negative breast cancer cells. Clin Cancer Res 18: 6634-6647, 2012.

7. Janjigian YY, Werner D, Pauligk C, Steinmetz K, Kelsen DP, Jäger E, Altmannsberger HM, Robinson E, Tafe LJ, Tang LH, et al: Prognosis of metastatic gastric and gastroesophageal junction cancer by HER2 status: A European and USA International collaborative analysis. Ann Oncol 23: 2656-2662, 2012.

8. Solit DB and Rosen N: Targeting HER2 in prostate cancer: Where to next? J Clin Oncol 25: 241-243, 2007.

9. Arteaga CL, Sliwkowski MX, Osborne CK, Perez EA, Puglisi F and Gianni L: Treatment of HER2-positive breast cancer: Current status and future perspectives. Nat Rev Clin Oncol 9: 16-32, 2011.

10. Kim JS, Kim HA, Seong MK, Seol H, Oh JS, Kim EK, Chang JW, Hwang SG and Noh WC: STAT3-survivin signaling mediates a poor response to radiotherapy in HER2-positive breast cancers. Oncotarget 7: 7055-7065, 2016.
11. Wang Y and Shang Y: Epigenetic control of epithelial-to-mesenchymal transition and cancer metastasis. Exp Cell Res 319: 160-169, 2013.

12. Rogers CD, Saxena A and Bronner ME: Sip1 mediates an E-cadherin-to-N-cadherin switch during cranial neural crest EMT. J Cell Biol 203: 835-847, 2013.

13. Płuciennik E, Nowakowska M, Pospiech K, Stępień A, Wołkowicz M, Gałdyszyńska M, Popęda M, WójcikKrowiranda K, Bieńkiewicz A and Bednarek AK: The role of WWOX tumor suppressor gene in the regulation of EMT process via regulation of CDH1-ZEB1-VIM expression in endometrial cancer. Int J Oncol 46: 2639-2648, 2015.

14. DeSantis C, Ma J, Bryan L and Jemal A: Breast cancer statistics, 2013. CA Cancer J Clin 64: 52-62, 2014.

15. Han JJ, Du BR and Zhang CH: Bioinformatic analysis of prognostic value of ARAP3 in breast cancer and the associated signaling pathways. Eur Rev Med Pharmacol Sci 21: 2405-2412, 2017.

16. De Maria R, Olivero M, Iussich S, Nakaichi M, Murata T, Biolatti B and Di Renzo MF: Spontaneous feline mammary carcinoma is a model of HER2 overexpressing poor prognosis human breast cancer. Cancer Res 65: 907-912, 2005.

17. Zhong BL, Bian LJ, Wang GM, Zhou YF, Chen YY and Peng F: Identification of key genes involved in HER2-positive breast cancer. Eur Rev Med Pharmacol Sci 20: 664-672, 2016.

18. Steffen AC, Göstring L, Tolmachev V, Palm S, Stenerlöw B and Carlsson J: Differences in radiosensitivity between three HER2 overexpressing cell lines. Eur J Nucl Med Mol Imaging 35: 1179-1191, 2008

19. Burk U, Schubert J, Wellner U, Schmalhofer O, Vincan E, Spaderna $S$ and Brabletz T: A reciprocal repression between ZEB1 and members of the miR-200 family promotes EMT and invasion in cancer cells. EMBO Rep 9: 582-589, 2008.

20. Vandewalle C, Comijn J, De Craene B, Vermassen P, Bruyneel E, Andersen H, Tulchinsky E, Van Roy F and Berx G: SIP1/ZEB2 induces EMT by repressing genes of different epithelial cell-cell junctions. Nucleic Acids Res 33: 6566-6578, 2005.

This work is licensed under a Creative Commons

Attribution-NonCommercial-NoDerivatives 4.0 International (CC BY-NC-ND 4.0) License. 